\title{
Calidad de vida, indicadores antropométricos y satisfacción corporal en un grupo de jóvenes colegiales \\ Quality of life, anthropometric indicators and body satisfaction in a group of high school students
}

\author{
Pedro Ureña Bonilla, Luis Blanco Romero, Jorge Salas Cabrera \\ Universidad Nacional de Costa Rica
}

\begin{abstract}
Resumen. El propósito es analizar la relación entre calidad de vida, indicadores antropométricos y satisfacción corporal en un grupo de estudiantes de secundaria. Participaron 196 estudiantes (78 hombres y 118 mujeres) de un colegio del cantón central herediano. Se midieron el peso, la talla, el porcentaje de grasa y el índice de masa corporal. La calidad de vida fue evaluada mediante el cuestionario Kiddokindl y la satisfacción corporal se determinó por medio de dos preguntas con una escala de respuesta tipo Likert. Se encontró correlaciones positivas y significativas entre satisfacción corporal y los dominios de la calidad de vida $(\mathrm{p}<0,05)$. El deseo de una figura corporal diferente se correlacionó negativamente con los dominios de calidad de vida $(\mathrm{p}<0,05)$. El ochenta y nueve por ciento de los jóvenes se sienten bien con sus cuerpos, aunque los hombres lo están más que las mujeres. La satisfacción corporal se relacionó negativamente con la forma corporal. Se encontró una relación inversa y significativa entre el peso, el índice de masa corporal y el deseo de otra figura corporal. Las relaciones encontradas entre satisfacción corporal, calidad de vida e indicadores antropométricos son consistentes con lo que se reporta en la literatura científica. Los resultados de este estudio evidencian la importancia que tiene para los jóvenes estar satisfechos con su cuerpo. No solo la estructura corporal objetiva es importante, sino también la valoración subjetiva del cuerpo, la cual está relacionada con la apreciación de la calidad de vida.
\end{abstract}

Palabras clave. Calidad de vida, antropometría, satisfacción corporal, jóvenes colegiales.

Abstract. To analyze the relationship between quality of life, anthropometric indicators and body satisfaction in a group of high school students. The study included 196 students (78 males and 118 females) of a central county school in Heredia. Measurements were made of weight, height, body fat percentage and body mass index. The quality of life was assessed by the KiddoKindl Questionnaire and body satisfaction was determined by two questions with a Likert response scale. Positive correlations were found between body satisfaction and different domains of quality of life ( $<<0.05)$. The desire of a different body shape was negatively correlated with the quality of life domains $(\mathrm{p}<0.05)$. Eighty nine percent of students feel good about their bodies, although males were more satisfied than females. Body satisfaction was negatively correlated with body shape. There was a significant inverse relationship between weight, BMI, and the desire of other body shape. The relationships between body satisfaction, quality of life and anthropometric indicators are similar to those reported in the literature. The results of this study show how important it is for young people to be satisfied with their bodies. In this sense, not only the objective body structure is essential for a good quality of life, but also the subjective perception of the body. Key words: Quality of Life, anthropometry, body satisfaction, high school students young college.

\section{Introducción}

La calidad de vida es un indicador importante de la salud y constituye un factor determinante para evaluar el impacto de los sistemas de salud (Müller, Lehamann, Küster \& Thyen, 2005; Urzua \& Mercado, 2008; Urzúa, Cortés, Prieto, Vega \& Tapia, 2009;). Una buena calidad de vida está íntimamente relacionada con la habilidad para desempeñarse con propiedad en actividades y contextos donde se presentan demandas físicas, psicológicas y sociales (Serra, 2006). En el caso de los adolescentes, se describen diferentes dominios que interactúan y se relacionan directamente con la calidad de vida, entre ellos: el bienestar corporal y psicológico, la autoestima, la familia, los amigos y la escuela (Serra, 2006). Especialmente en la adolescencia, los temas calidad de vida y satisfacción corporal juegan un papel central, sobre todo por tratarse de una fase de gran vulnerabilidad emocional en los y las jóvenes (Aguilar, Manrique, Tuesta \& Oblitas, 2010; Ramos, Rivera \& Moreno, 2010; Samperio y Del Barrio, 2011; Urzúa, Avendaño, Díaz \& Checura, 2010;), en la que pueden verse seriamente afectados la autoestima, el autoconcepto, la autoaceptación, el sentimiento de eficacia, la ansiedad y el estado anímico (Castro, Facio, Mollinedo \& Valdez, 2010; Ramos et al., 2010; Trejo,; Mazorra \& del Barrio, 2011). Durante la adolescencia se da una gran preocupación por la apariencia física, en razón de la presión social que ejercen los estándares de belleza y esbeltez (Mazorra \& del Barrio, 2011; Ramos et al., 2010; Sweeting, 2008). De hecho, la satisfacción con el propio cuerpo depende de la discrepancia que se autoperciba con respecto al modelo estético corporal establecido (Urzúa Bravo, Ogalde \& Vargas, 2011). Incluso en niños y niñas de 7 y 8 años de edad se reporta preocupación por la propia imagen corporal (Mancilla, Vásquez, Mancilla, Amaya \& Álvarez, 2012; Marcó, de Gracia, Trujano, Turro \& Olmo, 2004). De esta manera, se

Fecha recepción: 17-01-14- Fecha envío revisores: 09-05-14- Fecha de aceptación: 24-10-14 Jorge Salas Cabrera Josaca10@hotmail.com observa que la cultura juega un rol central en la conformación de la imagen corporal y de la satisfacción con el propio cuerpo (Mazorra \& del Barrio, 2011); consecuentemente, no estar a gusto con la propia figura corporal repercute tanto en el estado anímico de las personas, como en las relaciones interpersonales, afectando la calidad de vida (Trejos, et al., 2010).

La imagen corporal está estrechamente relacionada con el peso (Aguilar, et al., 2010), por lo que el sobrepeso y la obesidad se asocian positivamente con la insatisfacción corporal (Amaya, Álvarez \& Mancilla, 2010; Bully, Elousa \& López, 2012; Mazorra \& del Barrio, 2011; Trejos, et al., 2010;). En este contexto, se entiende la imagen corporal como la ilustración del tamaño, imagen y forma del cuerpo, vinculado a la dimensión emocional (Lopes de Sousa, 2008). Así, una imagen corporal distorsionada se relaciona con conductas desadaptativas como el distrés psicológico (baja auto-estima, imagen corporal pobre), lo cual predispone a las personas para la depresión crónica, los desórdenes alimentarios, el abuso de sustancias, los problemas afectivos y somáticos (Camacho, Fernández \& Rodríguez, 2006; Leone, et al., 2010;).

También se reporta relaciones significativas y negativas entreíndice de masa corporal y calidad de vida (Arif \& Rohrer, 2006; Kolotkin, et al., 2006; Modi, et al., 2008; Zeller \& Modi, 2006;), especialmente en los dominios que se relacionan con el funcionamiento físico, social y con el bienestar emocional. De manera particular, las mujeres con índices altos de masa corporal, tienden a reportar una peor calidad de vida (Modi, et al., 2008). En el caso de los adolescentes, los niveles de insatisfacción corporal son menores en hombres que en mujeres, lo que se explica principalmente por la influencia que tienen los pares en el desarrollo de la imagen corporal (Amaya, et al., 2010). La adolescencia, especialmente la femenina, es un período particularmente crítico, debido al conflicto que genera el choque entre el desarrollo puberal y el ideal estético, donde se sobrevalora el atractivo corporal (Camacho, Fernández \& Rodríguez, 2006). En este mismo sentido, Trejos et al. (2010), señalan que la persistencia de la insatisfacción corporal a lo largo de la adolescencia aumenta la probabilidad de desarrollar problemas 
alimentarios en el futuro (Anuel, Bracho, Brito, Rondón \& Sulbarán, 2012; Aleong, Duchesne \& Paus, 2007; Mazorra \& del Barrio, 2011;).

Por lo general, las mujeres adolescentes, en comparación con los varones, se muestran más preocupadas e insatisfechas con la imagen corporal (Murawski, Elizathe \& Rutsztein, 2011). De acuerdo con Cruz y Maganto (2003), el 63,9\% de las adolescentes manifiestan sentirse insatisfechas con su imagen corporal, mientras que menos del 50\% de los jóvenes reportan esa sensación. Asimismo, la proporción de varones que deseaba una figura más delgada es similar a la de quienes deseaban presentar una figura más robusta. Como contraste, el $90 \%$ de las mujeres que se sentían insatisfechas con su cuerpo, deseaban verse más delgadas (Murawski, et al., 2011). Mientras los muchachos se interesan por tener un cuerpo musculoso, las muchachas aspiran a tener cuerpos delgados que respondan al ideal de belleza instaurado por las presiones socioculturales, situación que puede convertirse en un factor determinante para el desarrollo de trastornos alimentarios (Anuel, et al., 2012; Mancilla, et al., 2012; Mazorra \& del Barrio, 2011;). Aunque también un porcentaje importante de hombres desea una figura más delgada y otros una figura más grande, en general, las mujeres comparadas con los hombres son más tendientes a considerarse obesas (Lopes de Sousa, 2008).

Estudios realizados por Trejos et al. (2010) reportaron un 17,7\% de adolescentes levemente insatisfechos con su imagen corporal y un 0,5\% con insatisfacción moderada. Asimismo, la prevalencia de sobrepeso y obesidad combinada fue de un 32\%. También se reportó correlaciones positivas y significativas entre el índice de masa corporal y la insatisfacción con la imagen corporal, esta última mayor en mujeres que hombres. No se encontraron diferencias significativas en cuanto a la insatisfacción corporal en función de la edad. Al seleccionar solo a los adolescentes con sobrepeso u obesidad y comparar la insatisfacción con el físico de acuerdo al sexo, se encontró que las mujeres están más insatisfechas. La comparación de los adolescentes según sexo, evidenció que en las mujeres, entre mayor sea el índice de masa corporal, mayor es la insatisfacción, contrario a los varones, donde las diferencias no fueron significativas. De esta manera, el índice de masa corporal se presenta como un factor asociado a la insatisfacción con la imagen corporal. Los adolescentes con mayor índice de masa corporal y de sexo femenino son los más susceptibles a sentirse insatisfechos (Trejos, et al., 2010). En España el porcentaje de adolescentes insatisfechas con su cuerpo es del $14,3 \%$, mientras que en adolescentes latinoamericanas asciende al $23 \%$ (Trejos, et al., 2010), llegando a un $40 \%$ en la población adolescente femenina norteamericana (Halliwell, Easun \& Harcourt, 2011).

También, Goldfield et al. (2010) reportan relaciones importantes entre imagen corporal y clasificación del peso. Los al comparar los obesos con los que tienen sobrepeso, los primeros reportan niveles superiores de insatisfacción corporal. Aunque los que tienen sobrepeso, evidencian mayor satisfacción corporal que los que tienen peso normal. Igualmente, los obesos reportan mayores síntomas represivos, anhedonia, autoestima negativa y mayores niveles de depresión que los jóvenes con sobrepeso y peso normal (Halliwell, et al., 2010). De esta forma, la insatisfacción corporal es una consecuencia adversa del sobrepeso y/o la obesidad y está relacionada con la calidad de vida.

La evidencia es clara en cuanto a la importancia que tiene el estudio de la calidad de vida en relación con indicadores antropométricos e imagen corporal, especialmente en la población de adolescentes, donde la vulnerabilidad emocional de los muchachos y muchachas es mucho mayor. Esta realidad obliga a las autoridades educativas y educadores en general a repensar e implementar estrategias de intervención, desde las posibilidades del movimiento humano, para promover la salud y coadyuvar en la formación integral de los jóvenes a nivel de secundaria. A pesar de la importancia de este tema, en Costa Rica apenas se estáincursionando en el análisis de esta problemática. Los resultados de este estudio servirán de base para impulsar la discusión sobre la salud del joven colegial desde una perspectiva integral, valorando tanto aspectos antropométricos como subjetivos relacionados con el bienestar de los jóvenes.
Sobre la base de la problemática que representa el tema en cuestión y a partir de la evidencia encontrada, el propósito de este estudio es describir y analizar la relación entre satisfacción corporal, variables antropométricas y calidad de vida en jóvenes colegiales costarricenses.

\section{Metodología}

\section{Tipo de estudio}

Se trató de un estudio de corte descriptivo-correlativo en el que de manera transversal se midió variables antropométricas y se registró información subjetiva sobre calidad de vida, imagen corporal y satisfacción con la imagen corporal.

\section{Participantes}

Se contó con un grupo de 196 estudiantes de secundaria, 78 hom-

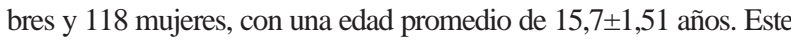
grupo fue definido a conveniencia de la institución por considerarse la forma en quemenos sealteraba el desarrollo de las actividades curriculares. Todos los jóvenes fueron informados del propósito del estudio y su participación fue voluntaria.

\section{Instrumentos}

La calidad de vida fue evaluada mediante el KiddoKindl, versión castellana desarrollada por Rajmil, Serra, Fernández, Aymerich, Cieza, Ferrer, Bullinger, (2004). Este instrumento contiene 24 preguntas distribuidas en seis dimensiones: bienestar físico (explora los niveles de actividad física, energía y estado físico), bienestar emocional (registra emociones positivas, satisfacción con la vida y sentimientos de equilibrio emocional), autoestima (orgullo, sensación de bienestar e ideas positivas), familia (relación con los padres, atmósfera de hogar), amigos (relación con otros adolescentes) y colegio (interés por la escuela, preocupación por notas y futuro). Las respuestas se recogen en una escala Likert de cinco categorías que oscilan desde 1 = nunca, hasta 5 = siempre. Entremayor sea la puntuación registrada mejor es la calidad de vida. El instrumento tiene una consistencia interna de uná $=0,70$ en el índice global y una estabilidad test retest que oscila en un rango de 0,52-0,80.

\section{Indicadores antropométricos}

Para determinar el peso y el porcentaje de grasa se utilizó un monitor de composición corporal marca Tanita (modelo BC554). El cálculo del índice de masa corporal se hizo mediante la fórmula IMC = peso/ talla². Como criterios de clasificación se utilizaron los siguientes percentiles: 5, para identificar bajo peso; 85 para sobrepeso y 95 para obesidad. La estatura se midió con una cinta métrica.

\section{La imagen corporal y la satisfacción con la figura corporal}

Esta información se recopiló mediante dos preguntas relacionadas con el nivel de satisfacción que experimentan los y las estudiantes en relación con el propio cuerpo (se usó una escala likert de 4 opciones, donde 1 equivale a poco satisfechos y 4 a muy satisfechos) y con el deseo de poseer otra forma o figura corporal (se utilizó una escala Likert de 5 puntos, donde 1 corresponde a la ausencia de deseo de otra figura corporal y 5 equivale al máximo deseo de otra forma corporal).

\section{Procedimiento}

Se estableció contacto con las autoridades de la institución educativa con el propósito de explicar el propósito y los alcances de la investigación. Durante dos días consecutivos se llevó a cabo las mediciones antropométricas y la aplicación de los cuestionarios. Los investigadores estuvieron siempre presentes para evacuar cualquier consulta por parte de los y las jóvenes encuestados.

\section{Análisis estadísticos}

Se calculó estadísticas descriptivas (medias y desviaciones estándar) e inferenciales por medio del Paquete Estadístico PASW 17.0, versión para Windows. Se realizaron pruebas de $\mathrm{Chi}^{2}$ para contrastar distribu- 
ciones y correlaciones producto momento de Pearson. Asimismo, se calcularon t student para comparar promedios en función del sexo de los participantes y ANOVAS para realizar comparaciones entre niveles. Todas las decisiones estadísticas se tomaron considerando el nivel á < .05 .

\section{Resultados}

El 50,5\% del estudiantado encuestado está satisfecho con su cuerpo, mientras que el $14,1 \%$ se manifiesta más o menos y poco satisfecho y el 35,4\% está muy satisfecho. Asimismo, el 33,9\% no desea tener una figura corporal diferente, pero el $51,5 \%$ desea poco una forma de cuerpo distinta y el 14,6\% desea mucho tener una figura diferente a la actual. Los hombres, comparados con las mujeres, manifiestan un mayor nivel de satisfacción con su cuerpo $\left(\mathrm{Chi}^{2}=11,35 ; \mathrm{p}<.01\right)$. En relación con la forma corporal, no se encontró diferencias significativas por sexo $\left(\mathrm{Chi}^{2}\right.$ $=1,55 ; \mathrm{p}>.05$ ).

El 62\% de los hombres tiene un peso normal, el 24\% bajo peso y el $14 \%$ evidencian sobrepeso y obesidad. En las mujeres, el $78 \%$ registra peso normal, el $8 \%$ bajo peso y el $10 \%$ sobrepeso y obesidad. No se registró relaciones importantes entre satisfacción corporal y peso (Chi ${ }^{2}$ $=2,90 ; \mathrm{p}>.05)$, tampoco entre el deseo de una forma corporal diferente y el peso $\left(\mathrm{Chi}^{2}=6,88 ; \mathrm{p}>.05\right)$. Igual fenómeno se dio al relacionar la satisfacción corporal con el índice de masa corporal $\left(\mathrm{Chi}^{2}=7,50 ; \mathrm{p}>\right.$ $.05)$ y con el deseo de una figura corporal diferente $\left(\mathrm{Chi}^{2}=8,71 ; \mathrm{p}>.05\right)$.

En relación con las medidas antropométricas, los hombres muestran una estatura y talla significativamente superior en comparación con las mujeres y, aunque el índice de masa corporal es similar para hombres y mujeres, el porcentaje de grasa es significativamente más alto en mujeres que en hombres (tabla 1 ).

Tabla 1.

Valores promedio (DT) para vari abl es antropométri cas y dominios del cons tructo calidad de vida en general y según sexo $(\mathrm{n}=196)$

\begin{tabular}{cclll} 
y segun sexo $(\mathrm{n}=196)$ & & & \\
\hline Variables & General & Hombres & Mujeres & Sig.
\end{tabular}

\begin{tabular}{lcccc}
\multicolumn{1}{c}{ Va riables } & General & Hombres & Mu jeres & Sig. \\
\hline Antropomérica & & & & \\
Peso & $58,26 \pm 12,79$ & $63,24 \pm 14,89$ & $54,66 \pm 9,61$ & 0.00 \\
Talla & $1,61 \pm 0,08$ & $1,68 \pm 0,07$ & $1,56 \pm 0,04$ & 0.00 \\
Porcentaje de grasa & $21,62 \pm 8,77$ & $15,65 \pm 7,30$ & $25,92 \pm 7,07$ & 0.00 \\
I.M.C.* & $22,17 \pm 3,99$ & $22,08 \pm 4,49$ & $22,23 \pm 3,60$ & 0.80 \\
Calidad de vida & & & & \\
Bienestar coporal & $3,61 \pm 0,74$ & $3,95 \pm 0,57$ & $3,39 \pm 0,75$ & 0.00 \\
Bienestar psicológico & $3,99 \pm 0,71$ & $4,06 \pm 0,63$ & $3,95 \pm 0,75$ & 0.29 \\
Autoestima & $3,93 \pm 0,83$ & $4,09 \pm 0,72$ & $3,82 \pm 0,88$ & 0.02 \\
Familia & $4,02 \pm 0,79$ & $4,10 \pm 0,74$ & $3,97 \pm 0,82$ & 0.24 \\
Amigos & $3,38 \pm 0,70$ & $3,84 \pm 0,71$ & $3,80 \pm 0,69$ & 0.71 \\
Escuela & $3,37 \pm 0,61$ & $3,43 \pm 0,60$ & $3,34 \pm 0,62$ & 0.28 \\
\hline *I.M.C.= Índice de masa corporal. & & & &
\end{tabular}

Por otro lado, los hombres muestran valores significativamente más altos en los dominios bienestar corporal y autovaloración en comparación con las mujeres (tabla 1 ).

El peso $(59,97 \pm 11,85)$ y el índice de masa muscular $(20,40 \pm 4,45)$ de los séptimos años comparados con el peso (62,66×12,76) e índice de masa muscular $(23,39 \pm 3,82)$ de los undécimos, mostró valores significativamente menores $(p<.05)$. Asimismo, en cuanto al dominio «Escuela» correspondiente al constructo calidad de vida, los séptimos $(3,69 \pm 0,58)$ reportan sentirse significativamente mejor en comparación con los undécimos $(3,19 \pm 0,53)$.

Lasatisfacción corporal se correlacionó positiva y significativamente con los dominios bienestar corporal $(r=0,31 ; \mathrm{p}<.01)$, bienestar psicológico $(r=0,40 ; p<.01)$, autoestima $(r=0,51 ; p<.01)$, familia $(r=0,10$; $\mathrm{p}>$.05) y escuela $(\mathrm{r}=0,23 ; \mathrm{p}<.01)$. Asimismo, la satisfacción con el propio cuerpo se correlacionó de forma significativa pero negativamente con el porcentaje de grasa $(r=-0,20 ; p<.01)$. En contraste, el deseo de otra forma corporal se correlacionó de manera significativa pero negativamente con los dominios bienestar corporal $(r=-0,17 ; p<.01)$, bienestar psicológico $(r=-0,31 ; p<.01)$, autoestima $(r=-0,48 ; p<.01)$, familia $(r=-0,24 ; p<.01)$, amigos $(r=-0,01 ; p>.05)$ y escuela $(r=$ $0,18 ; p<.01)$. También, el deseo de otra forma corporal se correlacionó significativa y positivamente con el peso $(r=0,18$; $p<.01)$ y con el índice de masa corporal $(r=0,18 ; p<.01)$. Entre los valores antropométricos y los dominios correspondientes a calidad de vida se registró correlaciones significativas y positivas entre bienestar corporal $\mathrm{y}$ talla $(r=0,15 ; p<.05)$, pero negativas y significativas con el porcentaje de grasa $(r=-0,22 ; p<.01)$.

\section{Discusión}

A pesar de que es un porcentaje pequeño de encuestados el que manifiesta cierta inconformidad con su figura corporal, es importante prestarle atención a ese grupo de jóvenes, especialmente por la estrecha relación reportada entre los trastornos alimentarios (anorexia y bulimia) y la insatisfacción con el propio cuerpo (Marks, Murray, Evans, Willing \& Sykes, 2008; Straub, 2007). En este sentido, se observa coincidencia en términos porcentuales entre los que no están muy satisfechos con su cuerpo (14,1\%) y los que desean una figura corporal diferente (14,6\%). Llama la atención el hecho de que las mujeres, comparadas con los hombres, manifiestan mayores niveles de insatisfacción con la figura corporal y con sus cuerpos. Esto podría considerarse como evidencia de que, en el caso de las mujeres, el ideal de belleza dictado por la sociedad ejerce una mayor influencia en la autoimagen corporal(Brannon \& Feist, 2010; Straub, 2007;). De acuerdo a los cánones de belleza, la sociedad demanda que las mujeres estén más atentas a esos estándares, situación que genera niveles de ansiedad y malestar en ellas. Esta realidad se asocia con el hecho de que la prevalencia de trastornos alimentarios es mucho mayor en la mujer que en el hombre (Marks, et al., 2008).

La distribución en categorías de acuerdo al índice de masa corporal fue similar para hombres y para mujeres; sin embargo, se registra un porcentaje importante de bajo peso en el caso de los hombres (24\%). Esta situación podría preocupar, por cuanto el bajo peso es indicador de déficit alimentario y este a su vez repercute en el crecimiento saludable de los y las jóvenes, y consecuentemente en las capacidades de rendimiento cognitivo a nivel de colegio. Los índices de obesidad y sobrepeso son inferiores a lo reportado en los últimos estudios publicados en esta materia (Encuesta Nacional de Nutrición 2008-2009; Estado de la Nación, 2012).

Como era de esperarse, por razones de tipo genético y fisiológico, los hombres evaluados son significativamente más altos y pesados que las mujeres (Powers \& Howley, 2007) y aunque el índice de masa corporal fue similar en ambos sexos, las mujeres, por razones fisiológicas (Powers \& Howley, 2007), mostraron porcentajes de grasa superiores a los hombres. Asimismo, como consecuencia de la edad y del crecimiento físico, los y las jóvenes de sétimo son más pequeños y con un índice de masa corporal menor que los undécimos.

En relación con el constructo calidad de vida, los datos registrados indican que el escenario en que mejor se sienten las personas encuestadas corresponde a la familia. Es decir, el seno familiar es considerado con un espacio de soporte no solo instrumental sino también psicológico. Esto es consistente con los argumentos expuestos por varios autores en cuanto al respaldo que se asocia con la familia, especialmente cuando se trata de jóvenes adolescentes, edad en la que contar con el apoyo familiar es fundamental (Martínez, Inglés, Piqueros \& Ramos, 2010; Martínez, Murgui, Musitu \& Monreal, 2007; Méndez \& Barva, 2008; Musitu \& Cava, 2003). Como contraste, las situaciones relacionadas con los amigos y con la escuela, son los escenarios que reciben la calificación más baja. Es posible que, por tratarse de adolescentes, las relaciones entre amigos están cargadas de conflicto, al vincularse con la búsqueda de identidad y deseo de reconocimiento (Martínez, et al., 2010). Por otro lado, aunque el paso por la escuela es una necesidad, también es cierto que la exigencia y disciplina que demanda la convierte en un espacio muchas veces complicado para los adolescentes (Martínez, et al., 2010), por lo que no es de extrañar que los jóvenes tiendan a sentirse a disgusto en ese escenario. 
Consistente con señalado párrafos atrás, las mujeres muestran un bienestar corporal más deteriorado en comparación con los hombres. De esta manera, la relación con el propio cuerpo representa para las mujeres todo un reto y esta situación puede asociarse no solo a la influencia que ejerce el ideal de belleza sobre ellas, sino también a los problemas vinculados con los cambios hormonales propios de esa fase de transición (Redondo \& Guevara, 2012). Muy relacionado con este tema, no es de extrañar que la autovaloración que hace la mujer sea peor a la de los hombres. Todo apunta a que la mujer tiene en esta etapa de la vida más problemas de autoestima (autovaloración) que el hombre (Castro, et al., 2012).

Por otro lado, los resultados evidenciaron que entre mayor sea la satisfacción corporal de los y las jóvenes encuestados, mejor es la valoración que hacen de su calidad de vida. Aunque se trate de una apreciación subjetiva, invertir en el propio cuerpo a través del ejercicio físico y de un control apropiado de la ingesta calórica de manera indirecta podría estar ejerciendo un efecto positivo sobre la imagen corporal y consecuentemente sobre la satisfacción corporal (Contreras, Gil, García Fernández \& Pastor, 2012); además de que esto se constituye en un antídoto para la incidencia de trastornos alimentarios(Hernández, Gómez \& Cuevas, 2012).

Fortaleciendo estos argumentos, los resultados también evidenciaron relaciones negativas entre el deseo de una nueva figura corporal y la calidad de vida. Es decir, entre menos desean los jóvenes una figura corporal diferente, mejor es la valoración subjetiva que hacen de su calidad de vida. Considerando que el tema relacionado con el cuerpo y la forma en que se percibe puede generar preocupaciones excesivas en los adolescentes, es esperable que cuando los jóvenes no están preocupados por su forma corporal, la valoración que hagan de su calidad de vida tenderá a ser mejor (Mancilla, et al., 2012; Rodríguez \& Cruz, 2008; Marcó, et al., 2004; Quinceno \& Vinacia, 2009; Wille, Erhart, Petersen \& Ravens, 2008). La importancia del cuerpo para el adolescente es indiscutible, de ello depende no solo su propia autoaceptación, sino también la aceptación de los demás (Anuel, et al., 2012; Arguello \& Romero, 2012; Mancilla, et al., 2012; Marcó, et al., 2004; Rodríguez \& Cruz, 2008). Quizá sentirse bien consigo mismo es el primer paso para que los demás los acepten. Trabajar fomentando la autoaceptación y el bienestar con el propio cuerpo es fundamental para el desarrollo integra del adolescente, especialmente en esa fase de transición, donde se consolida en gran parte la personalidad del joven (Inglés, 2009).

Como refuerzo de lo anterior, los resultados encontrados también evidenciaron que entre mayor sea el porcentaje de grasa de los jóvenes, menor es la satisfacción con el propio cuerpo. Nuevamente, el peso del ideal estético cultural puede estar jugando un rol importante (Mazorra \& del Barrio, 2011; Ramos, et al, 2010). Congruente con esa afirmación y como era de esperarse, a mayor talla mayor sensación de bienestar corporal, aunque entre mayor fue el porcentaje de grasa, peor fue la valoración del bienestar corporal.

\section{Conclusiones}

Los resultados reportados en este estudio evidencian la estrecha relación entre satisfacción corporal, calidad de vida e indicadores antropométricos como la estatura y el porcentaje de grasa corporal, aspectos que reciben una valoración sobredimensionada en el marco del modelo estético cultural imperante. Los datos recopilados subrayan la importancia que tiene considerar la valoración subjetiva de la imagen corporal y calidad de vida de los jóvenes a lo largo del proceso educativo en que están inmersos. En este contexto, el ejercicio físico regular y la educación en materia alimentaria son complementos indispensables para forjar estilos de vida saludables y para coadyuvar en la formación integral de los jóvenes.

\section{Referencias}

Aguilar, M., Manrique, L., Tuesta, M. \& Oblitas, M. (2010). Depresión y autoestima en adolescentes con obesidad y sobrepeso: un problema que pesa. Revista Enfermería Herediana, 3(1), 49-54.

Aleong, R, Duchesne, S \& Paus, T(2007). Current conceptualisation of body image dissatisfaction: have we got it wrong? Journal of Child Psychology and Psychiatry. 45(7), 1317-1325,

Amaya, A., Álvarez, R. \& Mancilla, J. (2010). Insatisfacción corporal en interacción con autoestima, influencia de pares y dieta restrictiva: Una revisión. Revista Méxicana de Trastornos Alimentarios, 1 , 76-89.

Anuel, A., Bracho, A., Brito, N., Rondón, E. \& Sulbarán, D. (2012). Autoaceptación y mecanismos cognitivos sobre la imagen corporal. Psicothema, 24(3), 390-395.

Arif, A., \& Rohrer, J. (2006). The relationship between obesity, hyperglycemia symptoms and health related quality of life among Hispanic and non Hispanic White children and adolescents. Biomedical Central Family Practice, 7(3), 1-7

Arguello, L. \& Romero, I. (2012). Trastorno de la imagen corporal. Revista Reduca (enfermería, fisioterapia y podología). Serie de trabajos de fin de grado. 4(1), 478-518.

Brannon, L. \& Feist, J. (2010). Health Psycology. An introduction to behavior and health. Sixt edition, Belmont, C.A: Wadsworth Cengace Learning(ISBN 0495090654)

Bully, P., Elousa, P. \& López, A. (2012). Insatisfacción corporal en la adolescencia: Evolución en una década. Anales de Psicología, 28(1), 196-202.

Camacho, K., Fernández, R. \& Rodríguez, I. (2006). Imagen corporal y práctica de actividad física en las chicas adolescentes: Incidencia de la modalidad deportiva. International Journal of Sport Science, 2(3), 1-19.

Castro, F., Ornelas, M., Cuadras, G, Guedea, J., Barrón, C. \& Hernández, R. (2012). Autoconcepto Físico en adolescentes: comparaciones por género. XV Congreso de Educación Física, Deporte y recreación. Facultad de Educación Física y Ciencias del Deporte. Universidad Autónoma de Chihuahua.

Contreras, O., Gil, P., García, L., Fernández, G. \& Pastor, J.C. (2012). Incidencia de un Programa de Educación Física en la percepción de la propia imagen corporal. Revista de Educación, 357, 281-303.

Cruz, S. \& Maganto, C. (2003). El test de las siluetas: un estudio exploratorio de la distorsión e insatisfacción con la imagen corporal en adolescentes. Revista del Instituto de Investigaciones de la Facultad de Psicología/UBA, 8(1), 79-99.

Goldfield, B., Henderson K., Buchholz, A., Obeid, N., \& Flament, MF(2010). Body dissatisfaction, dietary restrain, depression and weigth status in adolescents. Journal of school Health, 80(4), 18690

Programa Estado de la Nación. Informe Estado de la Nación 2009 [Internet]. Acceso: 20 de enero de 2012. Recuperado en: http:// w w w. p a h o . org / s a l u d e n la s a m e r i c a s / index.php?id=31\&option=com content

Halliwell, E, Easun, A \& Harcourt, D (2011). Body dissatisfaction: can a short media literacy message reduce negative media exposure effects amongst adolescent girls? Journal Health Psychologist. 2 (34), 396-403.

Hernández, A., Gómez, G. \& Cuervas, C. (2012). Relación entre dietaatracón y práctica de actividad física. Revista Psicología de la salud, 22(1), 99-106.

Inglés, C. J. (2009). Programa PEHIA: Enseñanza de Habilidades Interpersonales para adolescentes. Madrid: Pirámide.

Kolotkin, R., Zeller, M., Samsa, G, Polanichka, N., Yanovski, J., Maahs, D., González, D. \& Roehrig, H. (2006). Assessing weight-related quality of life in adolescents, obesity. National Library of Medicine, 14(3), 448-457.

Leone, J., Fetro, J., Kittleson, M., Welshimer, J., Partridge, J. \& Robertson, S. (2010). Predictors of Adolescent Male Body Image Dissatisfaction: Implications for Negative Health Practices and Consequences for School Health from a Regionally Representative Sample. Journal of School Health, 18(4), 174-184.

Lopes de Sousa, PM, (2008). Imagen corporal y obesidad en la adoles- 
cencia: estudio comparativo de aspectos sociodemográficos, psicológicos y conductuales. The Spanish Journal of Psychology,11(2), 551-5563

Mancilla, A., Vásquez, R., Mancilla, J., Amaya, A. \& Álvarez, G (2012). Insatisfacción corporal en niños preadolescentes: Una revisión sistemática. Revista Mexicana de Trastornos Alimentarios, 3, 62-79.

Marcó, M., de García, M., Trujano, P., Turro, O. \& Olmo, R. (2004). Imagen corporal, autoestima y actitudes alimentarias en preadolescentes. Revista de Psiquiatría. Compendio, 8(2). Recuperado de: http://www.psiquiatria.com/revistas/index.php/ psiquiatriacom/article/view/309

Marks, D., Murray, M., Evans, B., Willing, C. y Sykes, C. (2008). Psicología de la salud: teoría investigación y práctica. México: Manual Moderno.

Martínez, A., Inglés, C., Piqueros, J. \& Ramos, V. (2010). Importancia de los amigos y los padres en la salud y el rendimiento escolar. Journal of Research in Educational Psycholoy, 8(1), 111-138.

Martínez, B., Murgui, S., Musitu, G. \& Monreal, M. (2007). El rol del apoyo parental, las actitudes hacia la escuela y la autoestima en la violencia escolar en adolescentes. International Journal of Clinical and Health Psychology, 8(3), 679-692.

Méndez, P. \& Barva, E. (2008). Apoyo social percibido en adolescentes infractores de ley y no infractores. Psykhe, 17(1), 59-64.

Mazorra, S. \& del Barrio, J. (2011). La autoimagen en los adolescentes: Influencia de la cultura de la delgadez. Interpsiquis. $12^{\circ}$ Congreso virtual de psiquiatría: 1-9. Recuperado de: http:// www.psiquiatria.com/bibliopsiquis/handle/10401/1345/ browse?type=title\&sort_by=1\&order=ASC\&rpp=70\&etal=$1 \&$ starts_with $=\mathrm{L}$

Modi, A., Loux, T., Bell, S., Harmon, C., Inge, T. \& Zeller, M. (2008). Weight-specific health-related quality of life in adolescents with extreme obesity [Salud relacionada a la calidad de vida específica al peso en adolescentes con obesidad extrema]. Obesity a reserch journal, 16(10), 2266-2271. doi:10.1038/oby.2008.347

Müller, E., Lehamnn, H., Küster, R. M., \& Thylen, U. (2005). Lebensqualität und psychosoziale Anpassung bei Kindern und Jugendlichen mit juvenile idiopathischer Arthritis und reaktiven Arthritiden. Z. Rheumatol, 64(3), 177-187.

Murawski, B., Elizathe, L. \& Rutsztein, G. (2011). Hábitos alimentarios e insatisfacción con la imagen corporal. Un estudio comparativo entre mujeres y varones estudiantes de escuelas secundarias. Facultad de Psicología Universidad de Buenos Aires. I congreso internacional de investigación. Memoria del Congreso, 33-50.

Musitu, G. \& Cava, M. (2003). El rol del apoyo social en el ajuste de los adolescentes. Intervención psicosocial, 12(2), 179-192.

Powers, S. \& Howley, E. (2007). Exercise Physiology. Theory and application to fitness and performance. New York: McGraw Hill.
Quinceno, M. \& Vinaccia, S. (2009). Calidad de vida relacionada con la salud infantil: una aproximación conceptual. Psicología y Salud, 18(1), 37-44.

Rajmil, L., Serra-Sutton, V., Fernández, J.A. Berra, S., Aymerich, M., Cieza, A., Ferrer, M. Bullinger, M. \& Ravens-Sieberer (2004). Versión española del cuestionario alemán de calidad de vida relacionada con la salud en población infantil y de adolescentes: el Kindl. An pediatr, 60(6), 414-521.

Ramos, P., Rivera, F. \& Moreno, C. (2010). Diferencias de sexo en imagen corporal, control de peso e Índice de Masa Corporal de los adolescentes españoles. Psicothema, 22 (1), 77-83.

Redondo, J. \& Guevara, E. (2012). Diferencias de género en la prevalencia de la conducta prosocial y agresiva en adolescentes de dos colegios de la ciudad de Pasto - Colombia. Revista virtual Universidad Católica del Norte, 36, 173-192.

Rodríguez, S. \& Cruz, S. (2008). Insatisfacción corporal en adolescentes latinoamericanos y españoles. Psicothema, 20(1), 131-137.

Samperio, V. \& del Barrio, J. (2011). La autoimagen en los adolescentes: influencia de la cultura de la delgadez. $12^{\circ}$ Congreso Virtual de Psiquiatría y Neurociencia. Interpsiquis, 1-9.

Serra, V. (2006). Desarrollo de la versión española del Child Health and Illness Profile para medir el estado de salud percibido en la adolescencia. Universidad de Barcelona.

Straub, R. (2007). Health Psycology. Abiopsychosocial approach. New York: Worth Publishers.

Sweeting, H. (2008). Gendered dimensions of obesity in childhood and adolescence. Nutrition Journal, 7(1), 1-14.

Trejos, P., Castro, D., Facio, A., Mollinedo, F. \& Valdez, G. (2010). Insatisfacción con la imagen corporal asociada al índice de Masa Corporal en adolescentes. Revista. Cubana de Enfermería, 26(3), 144-154.

Urzúa,A., Avendaño, F., Diaz, S. \& Checura, D. (2010). Calidad de vida y conductas de riesgo en la preadolescencia. Revista Chilena Nutrición, 37(3), 282-292.

Urzúa, A., Bravo, M., Ogalde, M. \& Vargas, C. (2011). Factores vinculados a la calidad de vida en la adultez mayor. Revista Médica Chile, 139, 1006-1014.

Urzúa,A., Cortés, E., Prieto, L., Vega, S. \& Tapia, K. (2009). Autorreporte de la calidad de vida en niños y adolescentes escolarizados. Revista Chilena de Pediatría, 80(3), 238-244.

Urzúa,A. \& Mercado, G. (2008). La evaluación de la calidad de vida de los y las adolescentes a través del Kiddo-Kindl. Terapia psicológica, 26(1), 133-141.

Wille, N., Erhart, M., Petersen, Ch., \& Ravens, U. (2008). The impact of overweight and obesity on health related quality of life in childhood - results from an intervention study. Biomedical Central Public Health, 8(424), 1-9.

Zeller, M. H., \& Modi,A. C. (2006). Predictors of health related quality of life in obese youth. Obesity, 14, 122-130.
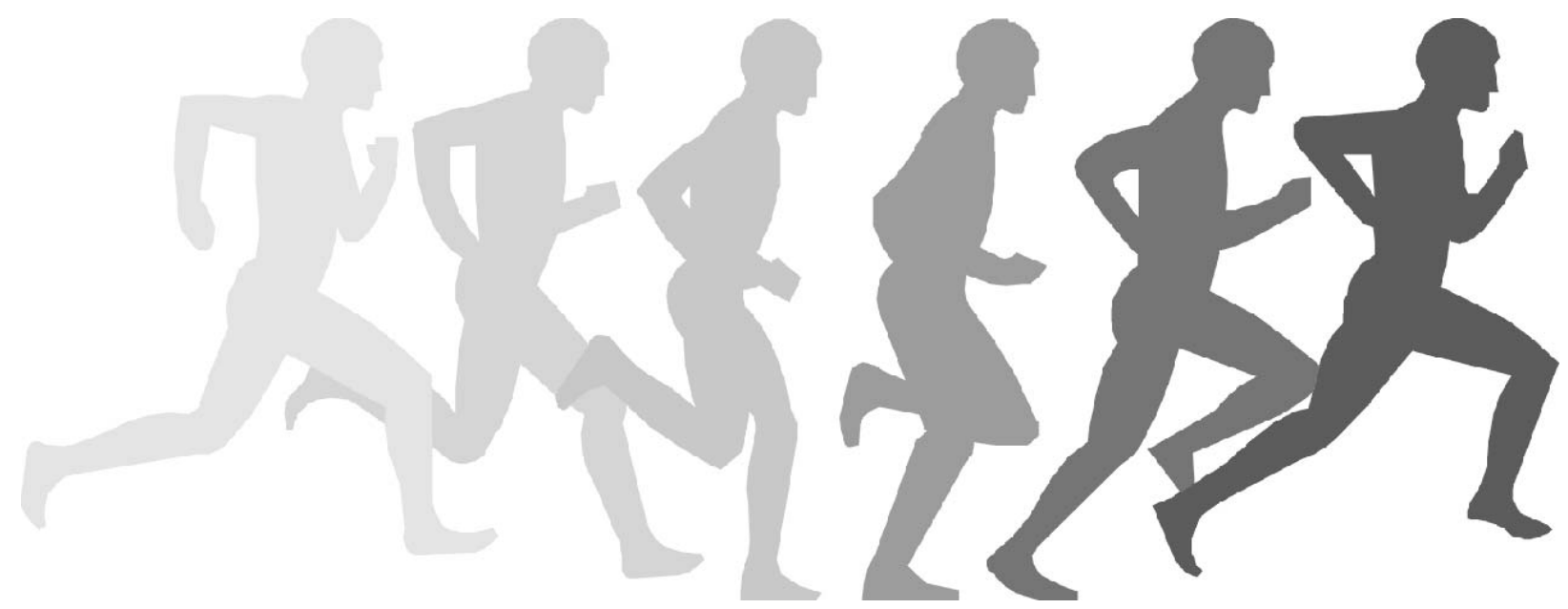